\title{
Study and Improvement for Slice Smoothness in Slicing Machine of Lotus Root
}

\author{
Deyong Yang ${ }^{1, *}$, Jianping $\mathrm{Hu}^{1}$, Enzhu Wei ${ }^{1}$, Hengqun $\mathrm{Lei}^{2}$, and Xiangci Kong ${ }^{2}$ \\ ${ }^{1}$ Key Laboratory of Modern Agricultural Equipment and Technology, \\ Ministry of Education \& Jiangsu Province, Jiangsu University, Zhenjiang, \\ Jiangsu Province, P.R. China 212013, \\ Tel.: +86-511-8; Fax: +86-511-8 \\ yangdy@163.com \\ 2 Jinhu Agricultural Mechanization Technology Extension Station, Jinhu county, \\ Jiangsu Province, P.R. China 211600
}

\begin{abstract}
Concerning the problem of the low cutting quality and the bevel edge in the piece of lotus root, the reason was analyzed and the method of improvement was to reduce the force in the vertical direction of link to knife. 3D parts and assemblies of cutting mechanism in slicing machine of lotus root were created under Pro/E circumstance. Based on virtual prototype technology, the kinematics and dynamics analysis of cutting mechanism was simulated with ADAMS software, the best slice of time that is $0.2 \mathrm{~s} \sim 0.3 \mathrm{~s}$ was obtained, and the curve of the force in the vertical direction of link to knife was obtained. The vertical force of knife was changed accordingly with the change of the offset distance of crank. Optimization results of the offset distance of crank showed the vertical force in slice time almost is zero when the offset distance of crank is $-80 \mathrm{~mm}$. Tests show that relative error of thickness of slicing is less than $10 \%$ after improved design, which is able to fully meet the technical requirements.
\end{abstract}

Keywords: lotus root, cutting mechanism, smoothness, optimization.

\section{Introduction}

China is a country of producing lotus root, lotus root system of semi-finished products of domestic consumption and external demand for exports is relatively large. In order to improve efficiency, reduce labor intensity, the group work, drawing on the principle of the artificial slice based on the design and development of a new type of lotus root slicer (Bi Wei and Hu Jianping, 2006). This new type of slicer solved easily broken cutting, stick knives, hard to clean up and other issues, but the process appears less smooth cutting, and some have a problem of hypotenuse piece of root. In this paper, analyzing cutting through the course of slice knife, the reasons causing hypotenuse was found, and the corresponding improvement of methods was proposed and was verified by the experiments.

\footnotetext{
${ }^{*}$ Corresponding author.
} 


\section{Structure of Cutting Mechanism of Slicing Machine}

Cutting mechanism of slice lotus root is the core of the machine, the performance of its direct impact on the quality of slice. Virtual prototyping of cutting mechanism of slice lotus root (Fig.1)was built by using Pro/E, and mechanism diagram of the body is shown in Fig.2. Cutting principle of lotus slicer adopted in the cardiac type of slider-crank mechanism was to add materials inside, which can be stacked several lotus root, lotus root to rely on the upper part of the self and the lower part of the lotus press down so that it arrives in the material under the surface of the baffle. While slider-crank mechanism was driven by motor, the knife installed on the slider cut lotus root. In the slice-cutting process it was found that parallelism of the surface at both ends of part of piece lotus was not enough, which can not meet the technical requirements for processing.

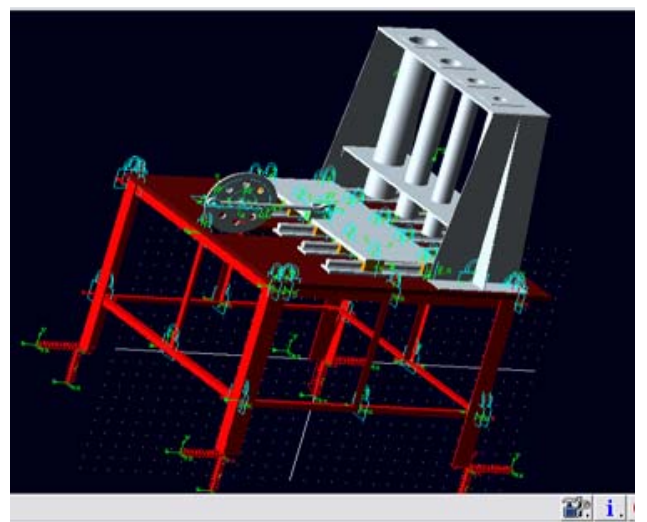

Fig. 1. Virtual prototyping of cutting mechanism

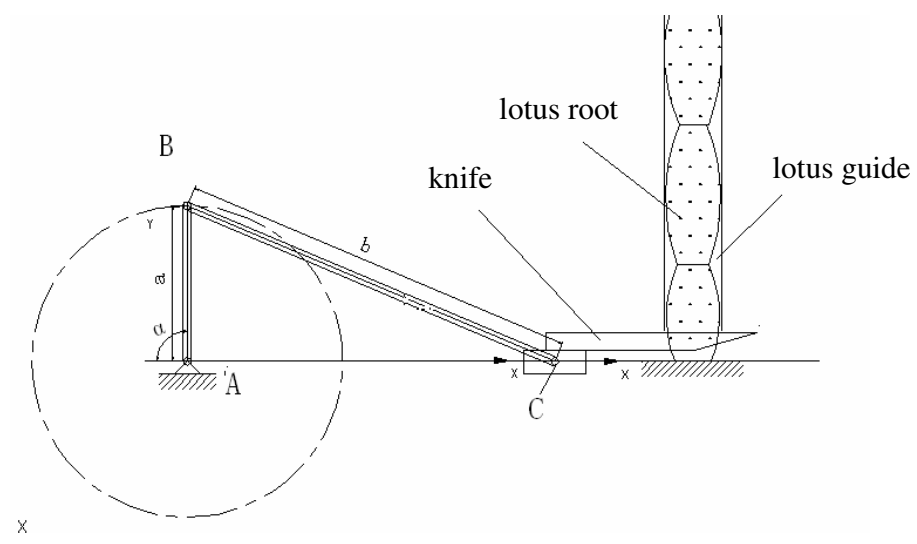

Fig. 2. Diagram of cutting mechanism 


\section{The Cause of the Bevel Edge}

Uneven thickness and bevel edge of cutting were related with forces on the slice knife in the process of cutting. In accordance with cutting mechanism ( Fig.2), without taking into account the friction and weight, the direction of force $\mathrm{F}$ of point $\mathrm{C}$ was along the link. Force $\mathrm{F}$ may be decomposed with a horizontal direction force component and a vertical direction force component. The horizontal force component pushed the knife moving for cutting, but the vertical force component caused the knife moving along the vertical direction. Because of the gap between the slider and the rail, the vertical force component made the blade deforming during the movement, and the knife could not move along the horizontal direction to cut lotus root, which caused the emergence of bevel edge. Thus, to reduce or eliminate the vertical force component in the cuttingchip was key to solve the problem of bevel edge and improve the quality of cutting.

When Crank speed was $60 \sim 90 \mathrm{r} / \mathrm{min}$, the horizontal and vertical direction of the force curve of point $\mathrm{C}$ connecting link and the blade hinge are shown in Fig. 3 and Fig.4 respectively. As can be seen from the chart, with the crank speed improvement the horizontal and vertical direction of the force in point $\mathrm{C}$ also increased. The horizontal force changed relatively stable during $0 \mathrm{~s} \sim 0.2 \mathrm{~s}$, which was conducive to cutting lotus, but the vertical force increased gradually. The more the vertical force was, the more detrimental to the quality cutting.

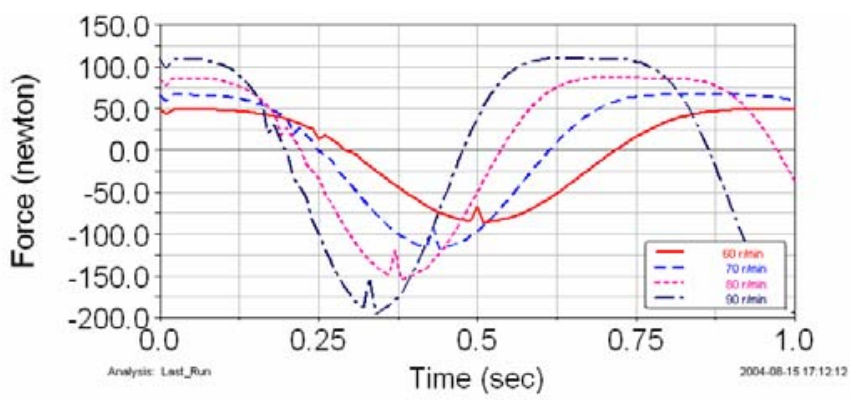

Fig. 3. Horizontal force of $\mathrm{C}$

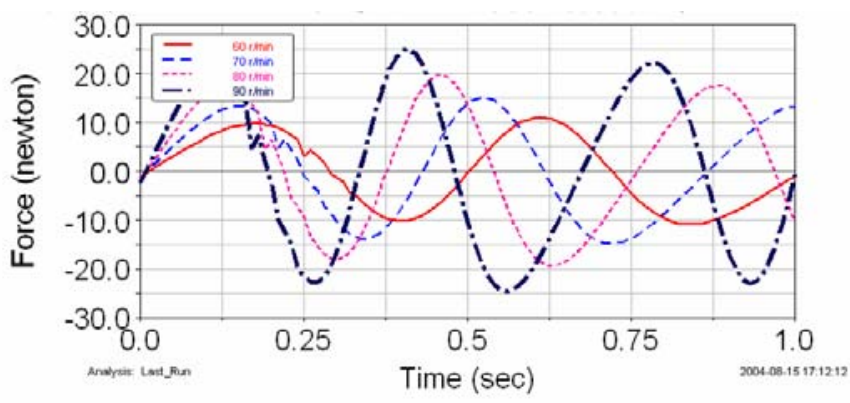

Fig. 4. Vertical force of $\mathrm{C}$ 


\section{Simulation and Optimization}

If improving flatness of the slicer, the structure was optimized to reduce the vertical force component, so as far as possible the level of cutting blade.

When crank speed was $60 \sim 90 \mathrm{r} / \mathrm{min}$ the velocity curve and acceleration curve of the knife center of mass are shown in Fig.5 and Fig.6 respectively. According to the speed curve, the speed of the knife center of mass was relatively large in a period of $0.2 \mathrm{~s} \sim$ $0.3 \mathrm{~s}$.In accordance with the requirements that the knife should have a higher speed during cutting lotus, so this period time was more advantageous to cutting than other terms. According to acceleration curve, when calculates by one cycle, the acceleration value was relatively quite small in the period of time, $0.15 \mathrm{~s} \sim 0.3 \mathrm{~s}$ compared with other time section, which indicated that the change of velocity was relatively small, simultaneously the force of inertia was small, and the influence of vibration caused by the force was small to the slicer. Therefore, this period of time, $0.2 \mathrm{~s} \sim 0.3 \mathrm{~s}$, to cut lotus root piece was advantageous in enhances the cutting quality of lotus root piece.

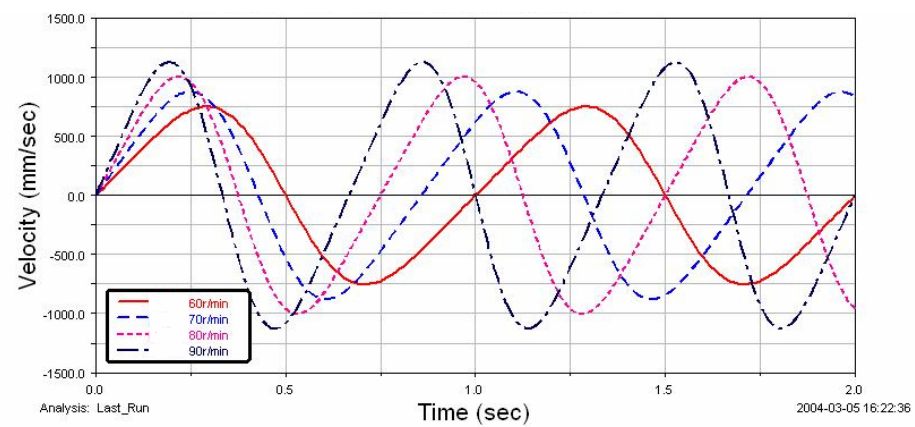

Fig. 5. Velocity curve of center of mass of knife

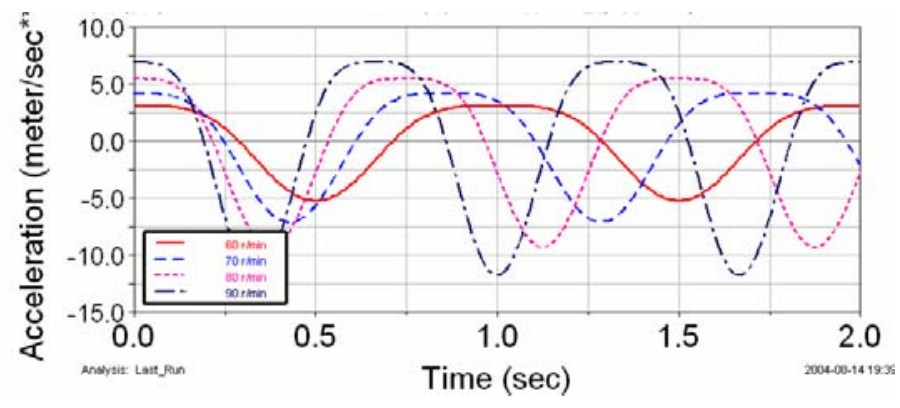

Fig. 6. Acceleration curve of center of mass of knife

Based on the above analysis, the vertical force component between link and the knife was the main reason for bevel edge. According to the characteristics of slidercrank mechanism, reducing the vertical force on the knife in the period of cutting time by altering crank offset was tried to enhance the quality of the cutting. When crank 
speed was $60 \mathrm{r} / \mathrm{min}$, the crank eccentricity was optimized. When the offset of the crank was $40 \mathrm{~mm}, 20 \mathrm{~mm}, 0 \mathrm{~mm},-20 \mathrm{~mm},-40 \mathrm{~mm},-60 \mathrm{~mm},-80 \mathrm{~mm},-120 \mathrm{~mm}$ respectively, the mechanism was simulated and the vertical force curves under different crank eccentricity were obtained, as shown in Fig 7.

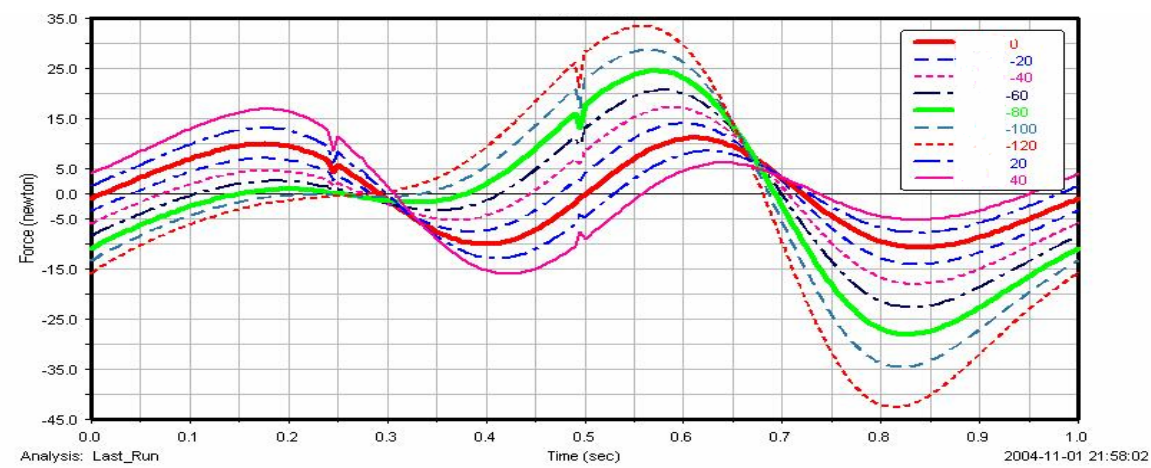

Fig. 7. Vertical force in different offset

Fig.7 indicates that: When the eccentricity was positive, the vertical force on point $\mathrm{C}$ increased gradually in $0.2 \mathrm{~s} \sim 0.3 \mathrm{~s}$ with the increase of crank offset; When the eccentricity was negative, the force decreased gradually first and then begun to increase along with the increase of crank offset. The force was almost zero when the eccentricity was $-80 \mathrm{~mm}$. So when the offset was $-80 \mathrm{~mm}$, the numerical of the force in $0.2 \mathrm{~s} \sim$ $0.3 \mathrm{~s}$ achieved the minimum and the quality of cutting was the best.

When the crank rotated in the other speed, there were the same optimization results. Fig. 8 shows the curve of vertical force in the offset of $0 \mathrm{~mm}$ and $-80 \mathrm{~mm}$ when the speed of crank was $80 \mathrm{r} / \mathrm{min}$. From the Fig 8 it is obvious that the vertical direction of the force of point $\mathrm{C}$ in $0.2 \sim 0.3 \mathrm{~s}$ reduced a lot when the eccentricity is $-80 \mathrm{~mm}$. Therefore, the vertical force could be reduced by optimizing the slider-crank mechanism of eccentricity.

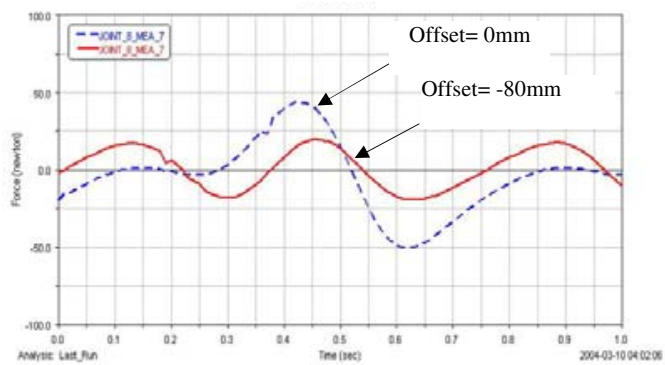

Fig. 8. Vertical force of C 


\section{Experimental Analysis}

The relative error of thickness of lotus root piece reflects the quality of cutting, which is generally controlled of $10 \%$. There always existed bevel edge phenomenon and the relative error of thickness was about $15 \%$ before structural optimization and improvement, which was difficult to meet the technical requirements. The offset in the slider-crank mechanism was optimized, and its structure was improved according to the results of optimization. After improvement cutting test were done in the conditions of crank speed for $80 \sim 110 \mathrm{r} / \mathrm{min}$ and statistical data about the relative error of thickness was shown in Table.1. Four levels were separated in the experiment, three times for each level.

Table 1. Relative error of thickness of slicing

\begin{tabular}{ccccc}
\hline \multirow{2}{*}{ NO } & \multicolumn{4}{c}{ Crank speed ( r/min) } \\
& 80 & 90 & 100 & 110 \\
\hline 1 & $6.6 \%$ & $6.4 \%$ & $8.2 \%$ & $9.5 \%$ \\
2 & $5.3 \%$ & $6.1 \%$ & $8.5 \%$ & $9.2 \%$ \\
3 & $6.4 \%$ & $7.9 \%$ & $7.9 \%$ & $9.4 \%$ \\
Average & $6.1 \%$ & $6.8 \%$ & $8.2 \%$ & $9.4 \%$ \\
\hline
\end{tabular}

It is derived from Table. 1 that the relative error of the thickness of slices could meet the technical indicators when the crank speed was $80 \sim 110 \mathrm{r} / \mathrm{min}$, especially in the crank rotation speed $80 \mathrm{r} / \mathrm{min}, 90 \mathrm{r} / \mathrm{min}$ the relative error of thickness was less than $7 \%$, and high quality was achieved.

\section{Conclusion}

The vertical force component acted on the knife in the process of cutting was the main reason for surface formation and bevel edge, so the key of improving the quality was to reduce the vertical force. Through slice knife velocity and acceleration simulation analysis the best time for slicing , $0.2 \mathrm{~s} \sim 0.3 \mathrm{~s}$, was obtained. By optimizing the offset of the crank the vertical force during cutting time was greatly reduced when the offset was $-80 \mathrm{~mm}$. Experiments were made after improving the design of lotus root slicer, which results showed that by changing the offset of the crank, the relative error of thickness could fully meet the requirements of less than $10 \%$. So the problem was basically solved that the flatness was not ideal and there was the issue of bevel edge.

\section{Acknowledgements}

This work was financially supported by Agricultural Mechanization in three agriculture projects, Jiangsu Province (NJ2007-17) and Tackling Key Problems of Science and Technology of Taizhou City (BE2008385). 


\section{References}

Wei, B., Jianping, H.: Study of Lotus Roots Slicing Techniques and Design of New Model. Journal of Agricultural Mechanization Research (12), 112-114 (2006) (in Chinese)

Enzhu, W.: The Simulation and Optimization on the new slicing machine of lotus root Based on Virtual Prototype Technology. Jiangsu University (2008) (in Chinese)

Ce, Z.: Mechanical Dynamics. Higher Education Press (1999)

Xiuning, C.: Optimal Design of Machinery. Zhejiang University Press (1999)

Liping, C., Yunqing, Z., Weiqun, R.: Dynamic Analysis of Mechanical Systems and Application Guide ADAMS. Tsinghua University Press, Beijing (2005) 